\title{
Matching Occluded Objects Invariant to Rotations, Translations, Reflections, and Scale Changes
}

\author{
Yasser El-Sonbaty ${ }^{1}$ and M.A. Ismail ${ }^{2}$ \\ 1 \\ Dept. of Computer Eng., Arab Academy for Sc. and Tech., Alexandria 1029, EGYPT \\ Dept. of Computer Sci., Faculty of Engineering, Alexandria 21544, EGYPT
}

\begin{abstract}
In this paper, a new algorithm for recognizing partially occluded objects is introduced. The proposed algorithm is based on searching for first three matched connected lines in both occluded and model objects, then left and right lines in both occluded and model objects are marked as matched lines as long as they have the same relations of distance ratio and angle to the last matched and connected lines. The process is repeated until there is no more three matched connected lines. The ratio_test is then performed to detect scattered matched points and lines. The new algorithm is invariant to translations, rotations, reflections and scale changes and has computational complexity of $O(m . n)$.
\end{abstract}

\section{Introduction}

Many algorithms have been reported in the literature for recognizing partially occluded objects using different methodologies such as point pattern matching, statistical pattern matching, syntactic methods, Fourier descriptors, graph matching and others [1].

The description of occluded objects and model objects can be done either by describing the object boundary with a sequence of control or dominant points or by using local features such as corners and holes and their relationship [2].

Liu and Srinath [3] developed a technique based on distance transformation in matching sequences of consecutive segments between a pair of contours. Lamdan et al. [4] improved the former technique by using the methodology of geometric hashing which is also used in [5]. Pikaz and Dinstein [6] presented an algorithm based on the total-curvature graph which is the direction of the first derivation of the curve with complexity $O(m . n)$. Tsang et al. [1] introduced a new scheme capable of combing the isolated clusters for object classification using three point matching and distance transformation. Price [7] suggested a method that builds a disparity matrix that contains the results of comparing the boundary segments of an object image with an occluded image. From this matrix the sequence of compatible segments is found. Bhanu and Faugeras [8] presented a stochastic labeling procedure that iterates in a hierarchical fashion until a criterion function is maximized. Han and Jang [9] used the 
concept of maximum curvature points in recognizing partially occluded objects using cliques for determining the compatible nodes with complexity $O\left(m^{2} \cdot n^{2}\right)$. Hong and Tan [10] proposed a method for transforming the dominant points to a canonical form and perform the matching between occluded and model objects by matching the canonical forms under Affine transformation. Neural networks was used in [11] with complexity $O\left(n^{3}\right)$. Koch and Kashyap [12] used vertex angles to create clusters corresponding to the object image, while, Bhanu and Ming [13] added the length of boundary segments to vertex angles to create disparity matrix and then formed clusters for the objects which involved in the occlusion. The concept of dynamic programming [14] was also used for recognizing occluded objects. The use of clustering approach was introduced in [15].

The core of this paper is to introduce a new algorithm for recognizing partially occluded objects based on searching for first three matched connected lines in both occluded and model objects, then left and right lines in both occluded and model objects are marked as matched as long as they have the same relation of distance ratio and angle to the last matched and connected lines. The process is repeated until there is no more matched connected three lines. The ratio_test is then performed to detect scattered matched points and lines. The main advantages of the proposed algorithm is being invariant to translations, rotations, reflections and scaling changes, also, having $O(m . n)$ as its computational complexity which is lower than many techniques reported in the literature. The results of applying the new algorithm show that it has higher percentage of recognition and lower processing time than techniques in [1], [5] and [6]. Section 2 describes the proposed algorithm and analyzes its computational complexity with comparison to other techniques. Experimental results are shown in section 3 . The conclusions about the performance of the new algorithm are introduced in section 4 .

\section{Recognizing Occluded Objects}

In this section, we show how to identify the objects constructing an occluded image. We start by showing how to extract the dominant points identifying the object boundary, followed by introducing the concept of transferring the connected lines into simple graphs, followed by the suggested procedure for matching these graphs, and finally the proposed algorithm along with its computational complexity are introduced.

\subsection{Extraction of Dominant Points}

Extraction of dominant points is also known as polygon approximation and many approaches have been reported in the literature for handling this problem. In this paper, we use the algorithm reported in [16]. The algorithm is divided into three stages based on the concept of perceptual organization. The first stage is low level linking and merging where points are grouped together based on the principles of proximity, similarity, and symmetry. Points are then linked together according to the concept of continuation. The second stage is smoothing that is used to suppress noise 
and delete visually insignificant points. The last stage is high level knowledge application. Detailed algorithm can be found in [16].

\subsection{Transforming Connected Lines to Graphs}

Given an image contains an unknown object Object $_{\text {occ }}$ that consists of some overlapped objects and a model object Object $_{\text {mod, }}$, where both objects are identified through a set of dominant points $P_{\text {occ }}$ and $P_{\text {mod }}$ respectively such that $\left|P_{\text {occ }}\right|=m$ and $\left|P_{\text {mod }}\right|=n$.

The algorithm starts by transforming every three connected lines in both Object $_{\text {occ }}$ and Object $_{\text {mod }}$ to simple graph as shown in Fig. 1, where $l_{\mathrm{i}}$ is the length of the line number $i$; i.e. connecting $i^{\text {th }}$ point to $i+1^{\text {th }}$ point. $\theta_{\text {i }}$ represents the angle between $i^{\text {th }}$ line and $i+1^{\text {th }}$ line. $l$ ' is the distance between end points of the three connected lines and $\theta^{\prime}$ is the angle between the $i^{\text {th }}$ line and the line connecting the end points. Every line from the three connected lines is represented by a node in the graph while edges represent the relation between connected nodes and have two attributes: 1- the ratio between the length of corresponding line to the length of main line $l_{i}, 2$ - the angle between corresponding connected nodes. It is clear that these attributes are invariant to translations, rotations, and scale changes.

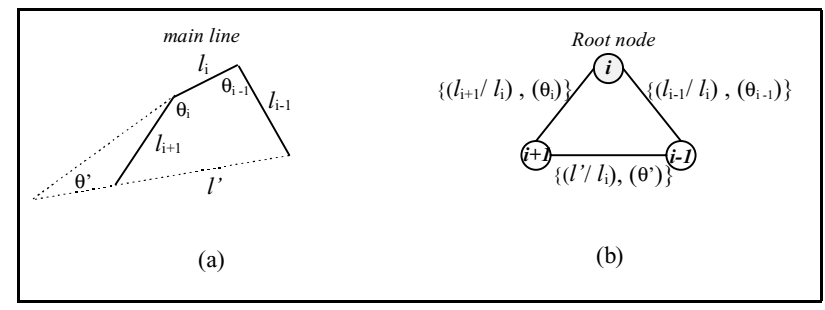

Fig. 1. Transforming from connected lines (a) to graph (b).

\subsection{Matching Lines-equivalent-Graphs}

After transforming every three connected lines in both Object $_{\mathrm{occ}}$ and Object $_{\text {mod }}$ to graphs, a matching process is then performed between these graphs to determine the matched lines. The matching process is done as follows:

Given two graphs $G_{\mathrm{occ}_{\mathrm{i}}}$ and $G_{\text {modj, }}$, where $1 \leq \mathrm{i} \leq m$ and $1 \leq \mathrm{j} \leq n$, as shown in Fig. 2, where $r, r^{\prime}, f$, and $f^{\prime}$ are the ratios between the length of lines to the length of main line as indicated by Fig. 1 , while $\theta, \theta^{\prime}, \phi$, and $\phi^{\prime}$ are the angles between the lines and the main line. 


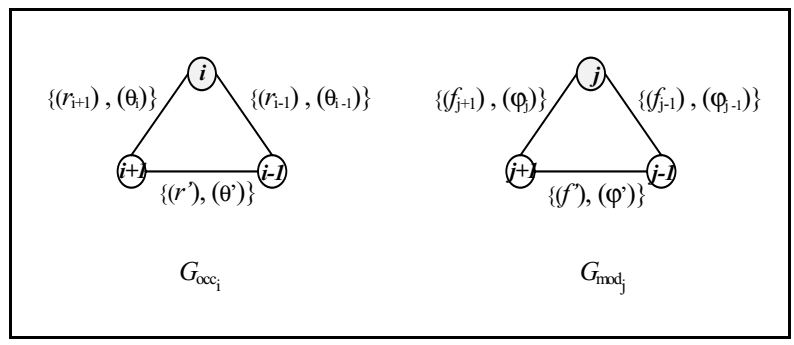

Fig. 2. Two graphs $G_{\text {occ }_{i}}$ and $G_{\text {modj }}$.

The distance between $G_{\mathrm{occ}_{\mathrm{i}}}$ and $G_{\text {modj }}$ is calculated as the minimum of the sum of the two diagonals of $2 \times 2$ matrix called node_to_node_matrix, where the entries in node_to_node_matrix are calculated as shown in Fig. 3, where $\operatorname{Abs}(x)$ returns the absolute value of $x$.

Formally the distance between $G_{\mathrm{occ}_{\mathrm{i}}}$ and $G_{\text {modj }}$ is calculated using the following equation:

$$
\begin{aligned}
\operatorname{dist}\left(G_{\mathrm{occ}_{\mathrm{i}}}, G_{\mathrm{mod} j}\right)=\min & \left\{\left(\text { node_to_node_matrix }_{\mathrm{i}+1, \mathrm{j}+1}+\text { node_to_node_matrix }_{\mathrm{i}-1, \mathrm{j}-1}\right),\right. \\
& \left.\left(\text { node_to_node_matrix }{ }_{\mathrm{i}+1, \mathrm{j}-1}+\text { node_to_node_matrix }_{\mathrm{i}-1, \mathrm{j}+1}\right)\right\} \\
& +\left(\operatorname{Abs}\left(r^{\prime}-f^{\prime}\right)+\operatorname{Abs}\left(\theta^{\prime}-\varphi^{\prime}\right)\right)
\end{aligned}
$$

If the sum of the main diagonal of the node_to_node_matrix is less than the sum of the secondary diagonal, then $i+1^{\text {th }}$ and $i-1^{\text {th }}$ lines in the occluded image is much probably matched with $j+1^{\text {th }}$ and $j-1^{\text {th }}$ lines respectively in the model object. On the other hand, if the sum of the secondary diagonal is less, then $i+1^{\text {th }}$ and $i-1^{\text {th }}$ lines in the occluded image is much probably matched with $j-1^{\text {th }}$ and $j+l^{\text {th }}$ lines respectively in the model object.

\subsection{Matching Process}

The algorithm proceeds matching three connected lines from the model object with all three connected lines in the occluded image through the matching of their equivalent graphs as previously discussed in section 2-2, and checks for every matching if it satisfies the requirements of accepting this matching as correct matching or not via equation 2 .

$$
\operatorname{dist}\left(G_{\text {occ }_{i}}, G_{\text {modj }}\right) \leq t_{\text {match }}
$$

where $t_{\text {match }}$ is some threshold indicating the tolerance expected in considering $G_{\mathrm{occ}_{\mathrm{i}}}$ and $G_{\text {modj }}$ as correctly matched and its value is dependent on some factors like noise level and distortions.

Once a correct matching between $G_{\mathrm{occ}_{\mathrm{i}}}$ and $G_{\text {modj }}$ is found, lines number $(i-1, i$, $i+1)$ in occluded image and $(j-1, j, j+1)$ in model objects are marked as matched lines and consequently, their equivalent points number $(i-1, i, i+1, i+2)$ and $(j-1, j, j+1$, $j+2)$ are also marked as matched points. The correct mapping between $(i-1, i, i+1)$ 
and $(j-1, j, j+1)$ can be easily clarified by knowing which diagonal has less sum in the node_to_node_matrix, Fig. 4 shows the available alternatives for this mapping.

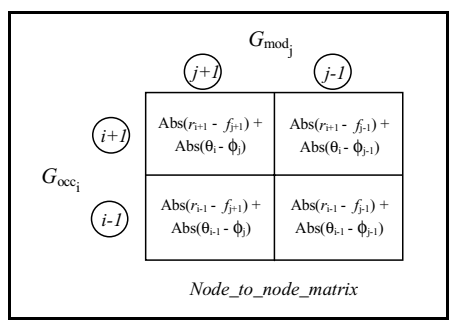

Fig. 3. node_to_node_matrix

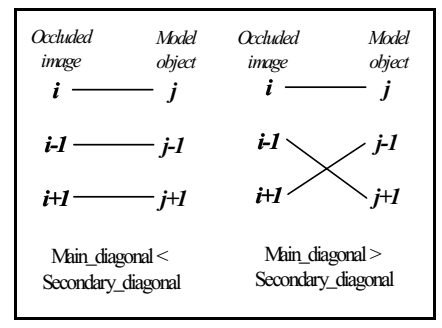

Fig. 4. Mapping between matched lines

From the mapping, the algorithm determines the next and previous connected lines to matched lines in both occluded and model objects, say $l_{\text {occ_next }}, l_{\text {occ_prev }}, l_{\text {mod_next }}$, and $l_{\text {mod_prev. }}$

Assume the following mapping $(i \rightarrow j),(i-1 \rightarrow j-1)$, and $(i+1 \rightarrow j+1)$, the algorithm examines if $l_{\text {occ_next }}$ and $l_{\text {mod_next }}$ are correctly matched by checking if the ratio and the angle between $l_{\text {occ_next }}$ and $j+l^{\text {th }}$ line in occluded image and the ratio and the angle between $l_{\text {mod_next }}$ and $i+1^{\text {th }}$ line in model object satisfy equations 3 and 4 .

$$
\begin{aligned}
& \operatorname{Abs}\left(\operatorname{angle}\left(l_{\text {occ_next }}, j+l^{\text {th }} \operatorname{line}_{\text {occ }}\right)-\operatorname{angle}\left(l_{\text {mod_next }}, i+l^{\text {th }} \operatorname{line}_{\text {mod }}\right)\right) \leq t_{\text {angle }} \\
& \operatorname{Abs}\left(\operatorname{ratio}\left(l_{\text {occ_next }}, j+l^{\text {th }} \operatorname{line}_{\text {occ }}\right)-\operatorname{ratio}\left(l_{\text {mod_next }}, i+l^{\text {th }} \text { line }_{\text {mod }}\right)\right) \leq t_{\text {ratio }}
\end{aligned}
$$

where angle $(x, y)$ returns the angle between the lines $x$ and $y$, and $\operatorname{ratio}(x, y)$ returns the ratio between the length of lines $x$ and $y . t_{\text {angle }}$ and $t_{\text {ratio }}$ are some threshold for accepting the difference in angle and ratio between $l_{\text {occ_next }}$ and $l_{\text {mod_next }}$ respectively.

In testing equations 3 and 4 we may get one of the following four cases:

1 - If equations 3 and 4 are both satisfied, then $l_{\text {occ_next }}$ and $l_{\text {mod_next }}$ and their equivalent points are marked as matched lines and points and the algorithm locates their new next connected lines and repeat checking their matching by testing equations 3 and 4 and replacing $j+1^{\text {th }}$ line occ $_{\text {o }}$ by old $l_{\text {occ_next }}$ and $i+1^{\text {th }}$ line $_{\text {mod }}$ by old $l_{\text {mod_next }}$.

2- If equation 3 and 4 are both violated, then the algorithm terminates the search in this direction and repeats testing equations 3 and 4 in the direction of $l_{\text {occ_prev }}$, and $l_{\text {mod_prev }}$ and if both directions were already tested then the algorithm starts again searching for three matched connected lines after excluding all matched lines in both occluded and model objects.

3- If equation 3 is correct while equation 4 is not, then it means that $l_{\text {occ_next }}$ and $l_{\text {mod_next }}$ are matched but the line $l_{\text {occ_next }}$ was cut by another line due to the overlapping between the objects, so $l_{\text {occ_next }}$ is only marked as matched line with holding the information that it matches the line $l_{\text {mod_next }}$. The process of marking $l_{\text {occ_next }}$ is done to reduce the search space in the occluded object. After that, the algorithm moves the search to the other direction as in step 2.

4- If equation 4 is correct while 3 is not, then the algorithm terminates searching in this direction and moves to the other direction as in step 2. 
The algorithm continues searching for three matched connected lines by checking equations 3 and 4 until there is no more three matched lines, then the algorithm switches to the procedure of ratio_test.

It is clear from the behavior of the matching process of the proposed algorithm that it is invariant to translations, rotations, reflections and scale changes.

\subsection{Ratio_test}

If a correct matching is encountered, then the algorithm detects that there are some common parts between occluded and model objects and so far only connected lines with minimum three lines were caught by the algorithm but no scattered points or lines could be observed and this is the main function of the procedure ratio_test.

The procedure ratio_test starts by locating any two matched lines in occluded and model objects that were previously matched correctly and coming from the satisfaction of equations 2 or both 3 and 4 . Assume these two lines are $l_{\text {occ }_{i}}$ and $l_{\text {modj }}$. To declare any unmatched two points $\left(x_{\mathrm{occ}_{\mathrm{k}}}, y_{\text {occ }_{\mathrm{k}}}\right)$ and $\left(x_{\text {mod }_{\mathrm{p}}}, y_{\text {mod }_{\mathrm{p}}}\right)$ in occluded and model objects as correctly matched points, they must satisfy equation 5 where $t_{\text {point }}$ is some threshold for accepting these points as a matched points. $l_{\mathrm{k}}$ and $l_{\mathrm{p}}$, are the length of the lines connecting tested points to the center of $l_{\text {occ }}$ and $l_{\text {mod }_{\mathrm{j}}}$ respectively, while $\theta_{\mathrm{k}}$ and $\varphi_{\mathrm{p}}$ are the angles between tested points and matched lines.

$$
\operatorname{Abs}\left(\theta_{k}-\varphi_{p}\right)+\operatorname{Abs}\left(\frac{l_{k}}{l_{o c c_{i}}}-\frac{l_{p}^{\prime}}{l_{\bmod j}}\right) \leq t_{\text {point }}
$$

Any two points in occluded and model objects satisfying equation 5 are marked as matched points. When two points are marked as matched points using the ratio_test, the algorithm checks the angles and the ratios of the two lines forming these matched points in both occluded and model objects, and if they are within certain prespecified tolerance, then the algorithm marked the two lines intersected at each point as matched lines.

\subsection{Analysis of Computational Complexity}

It is clear that the algorithm is divided into two parts. 1- searching for matched lines and has computational complexity $O(m . n)$, where $m$ and $n$ are number of points in occluded and model objects respectively, and 2- searching for scattered matched points using ratio_test and this part has also computational complexity of $O(m . n)$.

In summary, the computational complexity of the proposed algorithm is $O(m . n)$.

\section{Experimental Results}

In this section, the new algorithm is tested using some examples that show its efficiency and capability of handling the problem of recognizing partially occluded objects in different environments. 
Fig. 5 shows three examples of occluded objects with different scales, rotations, reflections and translations. The database library contains 12 model objects of different tools. Figures 5 (a) - (c) introduce the real images and figures 5 (d) - (f) show the corresponding extracted outer counters of these images respectively.

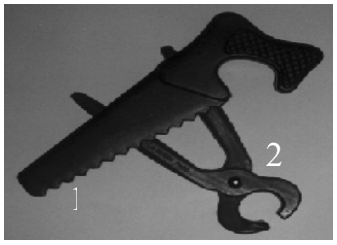

(a)

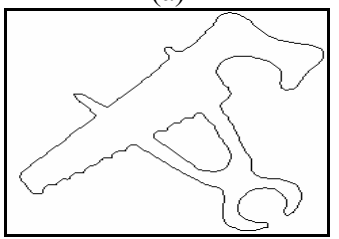

(d)

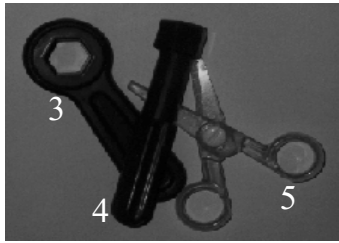

(b)

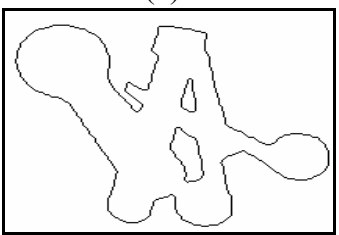

(e)

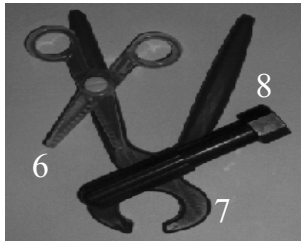

(c)

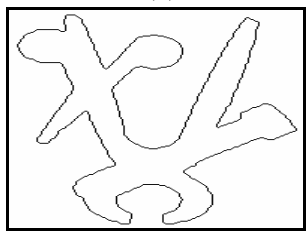

(f)

Fig. 5. (a), (b) and (c) Occluded objects; (d), (e) and (f) extracted counters.

Results of recognizing these occluded objects using the proposed algorithm in comparison with the algorithms of 3 point matching [1], local association [5], and total curvature function [6] are shown in tables I and II. Tables I shows the percentage of correct point matching for the occluded objects in Fig. 5 (a) - (c) respectively, while table II shows the processing time $(\mathrm{sec})$ taken for each algorithm to recognize all the model objects constructing each occluded object.

From tables I and II, it can be concluded that the proposed algorithm has higher recognition rate and lower processing time than algorithms of [1], [5] and [6].

\begin{tabular}{l|cc|ccc|ccc}
\hline & \multicolumn{2}{|c|}{ Fig. 5-(a) } & \multicolumn{3}{c|}{ Fig. 5-(b) } & \multicolumn{3}{c}{ Fig. 5 - (c) } \\
\hline \multicolumn{1}{c|}{ Tool \# $\rightarrow$} & $\mathbf{1}$ & $\mathbf{2}$ & $\mathbf{3}$ & $\mathbf{4}$ & $\mathbf{5}$ & $\mathbf{6}$ & $\mathbf{7}$ & $\mathbf{8}$ \\
\hline Proposed Algorithm & 97 & 98 & 96 & 95 & 98 & 83 & 97 & 96 \\
3Point matching [1] & 94 & 92 & 93 & 85 & 93 & 66 & 87 & 91 \\
Local association [5] & 88 & 90 & 84 & 78 & 86 & 66 & 83 & 79 \\
Total curvature [6] & 92 & 91 & 84 & 85 & 90 & 66 & 87 & 88 \\
\hline
\end{tabular}

Table I. Experimental results of Fig. 5 .

\begin{tabular}{l|ccc}
\hline & Fig. 5-(a) & Fig. 5-(b) & Fig. 5-(c) \\
\hline Proposed Algorithm & 0.40 & 0.55 & 0.53 \\
3 Point matching [1] & 0.98 & 1.21 & 1.08 \\
Local association [5] & 1.10 & 1.49 & 1.29 \\
Total curvature [6] & 1.97 & 3.57 & 2.38 \\
\hline
\end{tabular}

Table II. Processing time (sec) for experiments in Fig. 5 (a) - (c). 


\section{Conclusions}

In this paper a new algorithm for recognizing occluded objects is introduced. The proposed algorithm is based on searching for first three matched connected lines in both occluded and model objects, then left and right lines in both occluded and model objects are marked as matched lines as long as they have the same relation of distance ratio and angle to the last matched and connected lines. The process is repeated until there is no more three matched connected lines. The ratio_test is then performed to detect scattered matched points and lines. From algorithm discussion and experimental results, it can be concluded that the algorithm can be efficiently used for recognizing partially occluded objects and is invariant to rotations, translations, reflections, and scale changes. The computational complexity of the algorithm is $O(m . n)$. From experimental results, the proposed algorithm has higher percentage of correct point matching and lower processing time than techniques in [1], [5] and [6] .

\section{References}

1. Tsang P., Yuen P., Lam F.: Classification of Partially Occluded Objects Using Three Point matching and Distance Transformation. Pattern Recognition (1994) Vol. 27, $27-40$.

2. Bir Bhanu, Ming J. C.: Recognition of Occluded Objects: A Cluster-Structure Algorithm. Pattern Recognition (1987) Vol. 20, No. 2, 199-211.

3. Liu H. C., Srinath M. D.: Partial Shape Classification Using Contour Matching in Distance Transformation. IEEE Trans. On PAMI (1990), Vol. 12, No. 11, 1072-1079.

4. Lamdan Y., Schwartz J. T., Wolfson H. J.: Affine Invariant Model-based Object Recognition. IEEE Trans. On Robotics Automn (1990), Vol 6, No. 5.

5. Sethi I., Ramesh N.: Local Association Based Recognition of Two Dimensional Objects. Machine Vision and Applications (1992.)

6. Pikaz, Dinstein I.: Matching of Partially Occluded Planer Curves. Pattern Recognition (1995), Vol. 28, No. 2, 199-209.

7. Price K.: Matching Closed Contours. Proc. IEEE Workshop on Computer Vision (1984) 130134.

8. Bhanu B., Faugeras O. D.: Shape Matching of Two Dimensional Objects. IEEE Trans. On PAMI (1984) Vol. 6, 137-156.

9. Min-Hong Han, Dongsig J.: The Use of Maximum Curvature Points for the Recognition of Partially Occluded Objects. Pattern Recognition (1990), Vol. 23, No. 1, 21-33.

10. Hong, Tan X.: A New Approach to Point Pattern Matching. Proc. $9^{\text {th }}$ Int. Conf. Pattern Recognition (1988) 82-84.

11. Kurita T., Takahashi T., Ikeda Y.: A Neural Network Classifier for Occluded Images. $16^{\text {th }}$ Int. Conf. on Pattern Recognition (2002) 45-48.

12. Koch M. W., Kashyap R. L., "Using Polygons to Recognize and Locate Partially Occluded Objects", IEEE Trans. On PAMI, Vol. 9, P. 483-494, 1987.

13. Bhanu B., Ming J. C.: Recognition of Occluded Objects: A Cluster Structure Algorithm. Pattern Recognition (1987), Vol. 20, 199-211.

14. Petrakis E. G. M., Diplaros A., Milios E.: Matching and Retrieval of Distorted and Occluded Shapes using Dynamic Programming. IEEE Trans. On PAMI (2002)1501-1516.

15. Chang S., Hsuan F., Hsu W., Wu G.: Fast Algorithm for Point Pattern Matching: Invariant to Translations, Rotations and Scale Changes. Pattern Recognition (1997) 311-320.

16. Hu J., Yan H.: Polygonal Approximation of Digital Curves Based on the Principles of Perceptual Organization. Pattern Recognition (1997) Vol. 30, No. 5, 701-718. 